Chirurg 2015 $\cdot 86: 288$

DOI 10.1007/s00104-015-3006-0

Online publiziert: 20. Februar 2015

c) Springer-Verlag Berlin Heidelberg 2015

F. Rauchfuß - U. Settmacher

Klinik für Allgemein-, Viszeral- und Gefäßchirurgie, Universitätsklinikum Jena

\title{
3-D-Rekonstruktion zur Resektionsplanung bei zentralen hepatozellulären Karzinomen
}

ve Leberfunktion, $a$-Fetoprotein, Tumorgröße und Anzahl der Tumorherde.

Die dreidimensionale Rekonstruktion stellte sowohl die Gallenwege als auch arterielle, portalvenöse und venöse Strukturen sowie die Tumoren dar und diente als Grundlage für die virtuelle Operationsplanung.

Die intraoperativen Parameter unterschieden sich nicht hinsichtlich der angewandten Operationsmethoden, dem intraoperativen Blutverlust und der Notwendigkeit der Transfusion. In der Gruppe, die präoperativ eine 3-D-Operationsplanung erhalten hatte, konnte eine etwas geringere Operationszeit (294 min vs. $324 \mathrm{~min}, \mathrm{p}=0,028)$ und eine seltenere Anwendung des Pringle-Manövers (51,7\% vs. $71,4 \%, p=0,029)$ verzeichnet werden. Eine Aussage über das Volumen des resezierten Lebergewebes findet sich in der Studie nicht, sodass eine Einordnung der möglichen parenchymsparenden Technik nicht getroffen werden kann.

Die Rate an postoperativen Majorkomplikationen (Clavien III-V) war in der Gruppe mit 3-D-Operationsplanung signifikant niedriger $(3,3 \%$ vs. $14,3 \%$, $\mathrm{p}=0,048)$. Interessanterweise war auch die Rate an Galleleckagen in der 3-D-Gruppe niedriger ( $n=1$ vs. $n=6$ ), wenngleich dies nicht signifikant war.

\section{Fazit}

Die heute übliche präoperative zweidimensionale Standarddiagnostik (Computertomographie, Magnetresonanztomographie) lässt sich problemlos in dreidimensionale Rekonstruktionen umwandeln. Diese 3-D-Techniken erlauben eine präoperative Visualisierung des Tumors mit Bezug zu allen wichtigen ligamentären Strukturen bzw. zu den drainierenden Lebervenen. Dies erleichtert die Planung parenchymsparender Resektionen und senkt die perioperative Komplikationsrate.

Zusammenfassend bleibt festzustellen, dass eine dreidimensionale präoperative Bildgebung die individualisierte Operationsplanung bei Patienten mit zentralen Lebertumoren verbessert. Diese Studie bezieht sich auf asiatische Verhältnisse, wo die Leberteilresektion auch bei zentralen Lebertumoren in Zirrhose einen höheren Stellenwert hat als in Mitteleuropa. Dennoch legen die Ergebnisse nahe, dass eine dreidimensionale Bildgebung zur Senkung einer perioperativen Morbidität beitragen kann.

\section{Korrespondenzadresse}

\section{PD Dr. F. Rauchfuß}

Klinik für Allgemein-, Viszeral- und Gefäßchirurgie, Universitätsklinikum Jena, Erlanger Allee 101, 07747 Jena falk.rauchfuss@med.uni-jena.de

Interessenkonflikt. F. Rauchfuß und U. Settmacher geben an, dass kein Interessenkonflikt besteht. 